Lateral instabilities of cubic autocatalytic reaction fronts in a constant electric field

Ágota Tóth, Dezső Horváth, and Wim van Saarloos

Citation: The Journal of Chemical Physics 111, 10964 (1999); doi: 10.1063/1.480459

View online: https://doi.org/10.1063/1.480459

View Table of Contents: http://aip.scitation.org/toc/jcp/111/24

Published by the American Institute of Physics

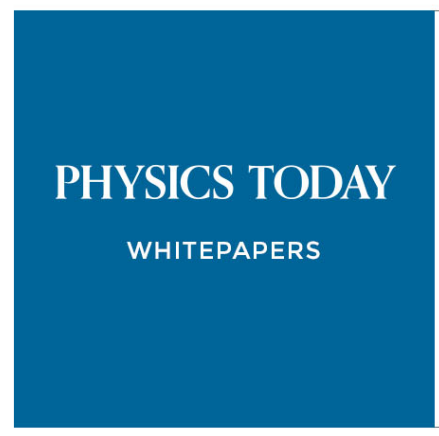
systems can do 


\title{
Lateral instabilities of cubic autocatalytic reaction fronts in a constant electric field
}

\author{
Ágota Tóth \\ Department of Physical Chemistry, József Attila University, P.O. Box 105, Szeged H-6701, Hungary \\ Dezső Horváth \\ Department of Physical Chemistry, József Attila University, P.O. Box 105, Szeged H-6701, Hungary \\ and Laboratory of Nonlinear Studies and Computation, Research Institute for Electronic Science, \\ Hokkaido University, Sapporo 060-0812, Japan \\ Wim van Saarloos \\ Instituut-Lorentz, Universiteit Leiden, Postbus 9506, 2300 RA Leiden, The Netherlands
}

(Received 4 August 1999; accepted 24 September 1999)

\begin{abstract}
The region of instability for planar reaction fronts of cubic autocatalysis between ionic species under constant electric field has been determined accurately. The ratio of diffusion coefficients at the onset of instability $\delta_{\text {cr }}$ is substantially varied by the component-dependent drift and directly proportional to the concentration of the autocatalyst behind the front $\beta_{s}$ as $\delta_{\mathrm{cr}}=2.3002 \beta_{s}$. This opens the possibility to use electric field as a control parameter for reaction-front instabilities. The dispersion relation calculated from the linear stability analysis of the full system is in good agreement with the initial evolution of the Fourier modes associated with the slightly perturbed planar reaction front obtained by the direct integration of the governing equations in two spatial dimensions. (c) 1999 American Institute of Physics. [S0021-9606(99)50148-7]
\end{abstract}

\section{INTRODUCTION}

Autocatalytic reaction-diffusion fronts with short-range activation may lose stability and result in cellular front structures. ${ }^{1}$ This diffusion-driven lateral instability has been studied thoroughly in cubic autocatalysis, ${ }^{2-4}$ which is the simplest chemical system exhibiting the phenomenon. Experimental studies have reported the existence of cellular structures evolving from unstable planar reaction fronts under isothermal conditions in the iodate-arsenous $\operatorname{acid}^{5}$ and the chlorite-tetrathionate reactions. ${ }^{6}$ The latter system has also been the subject of quantitative analysis ${ }^{7}$ and extension to three spatial dimensions. ${ }^{8}$

In reactions involving ionic species, externally imposed electric field may have a dramatic effect on the spatiotemporal behavior as a result of the component-dependent migration as observed in the excitable Belousov-Zhabotinsky reaction, ${ }^{9,10}$ one-dimensional propagating fronts of the iodate-arsenous acid reaction, ${ }^{11}$ and mosaic patterns of the methylene blue-sulfide-oxygen system. ${ }^{12}$ When the applied electric field is constant, the effect is similar to that of differential flow; ${ }^{13,14}$ a component-dependent drift arises with a constant velocity. Rovinsky et al. ${ }^{15}$ have investigated the stability of planar autocatalytic fronts in the presence of differential flow and found that a selective drift in the direction of front propagation either stabilizes or destabilizes the planar front structure near the onset of instability depending on the orientation of the flow. By applying a thin-reaction-front approximation, ${ }^{16}$ Horváth et al. ${ }^{17}$ have furthermore presented that destabilization is enhanced when the ionic migration tends to separate the components. The concentration of the autocatalyst behind the front has been shown to play an important role in the electric field induced lateral instability, which is not restricted to the neighborhood of the onset of instability.

In this work we carry out a linear stability analysis of the full two-dimensional system of cubic autocatalysis between ionic species under constant electric field in order to locate the region of lateral instability. The factors affecting the stability are stated and the onset of instability is determined accurately. One surprising finding, derived analytically, is that the critical ratio of diffusion coefficients $\delta_{\text {cr }}$ is linearly related to the concentration of the autocatalyst behind the front $\beta_{s}$. The dispersion relation is also calculated and compared to the temporal behavior of the Fourier modes in the front evolved from a slightly perturbed planar reaction front.

\section{GOVERNING EQUATIONS}

Under a constant electric field, reaction fronts of cubic autocatalysis are governed by

$$
\begin{aligned}
& \frac{\partial \alpha}{\partial \tau}=\delta \nabla^{2} \alpha-z_{A} \varepsilon \delta \frac{\partial \alpha}{\partial \xi}-\alpha \beta^{2}, \\
& \frac{\partial \beta}{\partial \tau}=\nabla^{2} \beta-z_{B} \varepsilon \frac{\partial \beta}{\partial \xi}+\alpha \beta^{2},
\end{aligned}
$$

where $\alpha$ and $\beta$ are the dimensionless concentrations of reac$\operatorname{tant} A$ with charge $z_{A}$ and autocatalyst $B$ with charge $z_{B}$ relative to the initial concentration of $A$ far ahead of the front and $\nabla^{2}=\partial^{2} / \partial \xi^{2}+\partial^{2} / \partial \eta^{2}$. The ratio of the diffusion coefficients is taken as $\delta=D_{A} / D_{B}$ and $\varepsilon$ represents the dimensionless electric field strength. For planar fronts, it is convenient to introduce a new coordinate $\zeta=\xi-c \tau$ that travels at the same velocity $c$ as the front, upon which the equations in Eq. (1) then become 


$$
\begin{aligned}
& 0=\delta \frac{d^{2} \alpha}{d \zeta^{2}}+\left(c-z_{A} \varepsilon \delta\right) \frac{d \alpha}{d \zeta}-\alpha \beta^{2}, \\
& 0=\frac{d^{2} \beta}{d \zeta^{2}}+\left(c-z_{B} \varepsilon\right) \frac{d \beta}{d \zeta}+\alpha \beta^{2},
\end{aligned}
$$

since in the new coordinate system $(\zeta, \eta, \tau), \partial \alpha / \partial \tau=0$, $\partial \alpha / \partial \eta=0$ and the same holds for $\beta$. The boundary conditions are given as $\alpha(+\infty) \rightarrow 1, \beta(+\infty) \rightarrow 0$ representing the reactant side ahead of the front and $\alpha(-\infty) \rightarrow 0, \beta(-\infty)$ $\rightarrow \beta_{s}$ the product side behind the front, while the concentration gradients vanish at both limits. The existence of a reaction front requires that both coefficients of the concentration gradients in Eq. (2) be positive, leading to an expression for the final concentration of the autocatalyst behind the front ${ }^{18}$ as

$$
\beta_{s}=\frac{c-z_{A} \varepsilon \delta}{c-z_{B} \varepsilon}=1+\frac{\Delta \mathrm{v}}{c-z_{B} \varepsilon} .
$$

In this equation, which can be simply obtained by adding Eqs. (2a) and (2b) and integrating between the limits, $\Delta \mathrm{v}$ $=\left(z_{B}-z_{A} \delta\right) \varepsilon$ is the difference in the drift velocity of the components relative to the reactant. In the case of unequal mobilities $\left(z_{B} \neq z_{A} \delta\right)$, therefore, the drift induced by electric field varies $\beta_{s}$ by increasing or decreasing the spatial overlap of the components.

\section{LINEAR STABILITY ANALYSIS OF PLANAR FRONTS}

For the stability analysis we first rescale the variables and coordinates according to $\widetilde{\alpha}=\alpha, \widetilde{\beta}=\beta / \beta_{s}, \widetilde{\zeta}=\zeta \sqrt{\beta_{s}}, \widetilde{\eta}$ $=\eta \sqrt{\beta_{s}}$, and $\tilde{\tau}=\tau \beta_{s}^{2}$ to obtain

$$
\begin{aligned}
& \frac{\partial \widetilde{\alpha}}{\partial \widetilde{\tau}}=\frac{\delta}{\beta_{s}} \widetilde{\nabla}^{2} \widetilde{\alpha}+\frac{Q_{0}}{\beta_{s}^{3 / 2}} \frac{\partial \widetilde{\alpha}}{\partial \widetilde{\zeta}}-\widetilde{\alpha} \widetilde{\beta}^{2}, \\
& \beta_{s} \frac{\partial \widetilde{\beta}}{\partial \widetilde{\tau}}=\widetilde{\nabla}^{2} \widetilde{\beta}+\frac{Q_{0}}{\beta_{s}^{3 / 2}} \frac{\partial \widetilde{\beta}}{\partial \widetilde{\zeta}}+\widetilde{\alpha} \widetilde{\beta}^{2},
\end{aligned}
$$

where $Q_{0}=c-z_{A} \varepsilon \delta$. A small spatial perturbation of the planar solution $\widetilde{\alpha}_{0}(\widetilde{\zeta}), \widetilde{\beta}_{0}(\widetilde{\zeta})$ is then introduced as

$$
\begin{aligned}
& \widetilde{\alpha}(\widetilde{\zeta}, \widetilde{\eta}, \widetilde{\tau})=\widetilde{\alpha}_{0}(\widetilde{\zeta})+\sum_{k \geqslant 0} \widetilde{\alpha}_{1, k}(\widetilde{\zeta}) \phi_{k}(\widetilde{\eta}, \widetilde{\tau}), \\
& \widetilde{\beta}(\widetilde{\zeta}, \widetilde{\eta}, \widetilde{\tau})=\widetilde{\beta}_{0}(\widetilde{\zeta})+\sum_{k \geqslant 0} \widetilde{\beta}_{1, k}(\widetilde{\zeta}) \phi_{k}(\widetilde{\eta}, \widetilde{\tau}),
\end{aligned}
$$

where $k$ is the wave number associated with the perturbation. After the substitution of Eq. (5) into Eq. (4) and linearization in $\phi_{k}$, the various spatial modes decouple. Taking then the form of $\exp (\omega \widetilde{\tau}+i k \widetilde{\eta})$, we obtain

$$
\left(\begin{array}{cc}
\omega+\delta^{\prime} k^{2} & 0 \\
0 & \beta_{s} \omega+k^{2}
\end{array}\right)\left(\begin{array}{c}
\widetilde{\alpha}_{1, k} \\
\widetilde{\beta}_{1, k}
\end{array}\right)=\hat{L}\left(\begin{array}{c}
\widetilde{\alpha}_{1, k} \\
\widetilde{\beta}_{1, k}
\end{array}\right),
$$

where matrix operator $\hat{L}$ is given as
$\hat{L}=\left(\begin{array}{cc}\delta^{\prime} \frac{\partial^{2}}{\partial \widetilde{\zeta}^{2}}+Q_{0}^{\prime} \frac{\partial}{\partial \widetilde{\zeta}}-\widetilde{\beta}_{0}^{2} & -2 \widetilde{\alpha}_{0} \widetilde{\beta}_{0} \\ \widetilde{\beta}_{0}^{2} & \frac{\partial^{2}}{\partial \widetilde{\zeta}^{2}}+Q_{0}^{\prime} \frac{\partial}{\partial \widetilde{\zeta}}+2 \widetilde{\alpha}_{0} \widetilde{\beta}_{0}\end{array}\right)$,

with $\delta^{\prime}=\delta / \beta_{s}$ and $Q_{0}^{\prime}=Q_{0} / \beta_{s}^{3 / 2}$. The planar front loses stability when $\omega$ becomes positive for spatial modes in the range of $0<k<k_{\max }$, representing a long-wavelength instability. ${ }^{19}$ The $k=0$ mode corresponds to the homogeneous translation in the direction of propagation

$$
\begin{aligned}
& \widetilde{\alpha}_{0}(\widetilde{\zeta}+d \widetilde{\zeta})=\widetilde{\alpha}_{0}(\widetilde{\zeta})+\frac{d \widetilde{\alpha}_{0}}{d \widetilde{\zeta}} d \widetilde{\zeta} \\
& \widetilde{\beta}_{0}(\widetilde{\zeta}+d \widetilde{\zeta})=\widetilde{\beta}_{0}(\widetilde{\zeta})+\frac{d \widetilde{\beta}_{0}}{d \widetilde{\zeta}} d \widetilde{\zeta},
\end{aligned}
$$

to which the planar solution is invariant. The translation mode $\left(d \widetilde{\alpha}_{0} / d \widetilde{\zeta}, d \widetilde{\beta}_{0} / d \widetilde{\zeta}\right)^{T}$ is a right zero mode of $\hat{L}$ as

$$
0=\hat{L}\left(\begin{array}{c}
\frac{d \widetilde{\alpha}_{0}}{d \widetilde{\zeta}} \\
\frac{d \widetilde{\beta}_{0}}{d \widetilde{\zeta}}
\end{array}\right)
$$

which is the scaled version of the linearized form of Eq. (2), and hence has $\omega=0$. By comparing Eqs. (5) and (8), we realize that $\widetilde{\alpha}_{1,0}$ and $\widetilde{\beta}_{1,0}$ are $d \widetilde{\alpha}_{0} / d \widetilde{\zeta}$ and $d \widetilde{\beta}_{0} / d \widetilde{\zeta}$, respectively, and that these indeed comprise the eigenvector belonging to the zero eigenvalue of $\hat{L}$.

The onset of lateral instability occurs when $d \omega / d\left(k^{2}\right)$ at $k=0$ changes its sign from negative to positive as some parameter is varied; we therefore investigate the behavior of the $\omega-k^{2}$ curve in the vicinity of the origin. When the wave number is slightly increased so that $k^{2}$ is of $O(\epsilon)$ with $\epsilon$ being an arbitrarily small positive number, $\omega$ takes on a value of $O(\epsilon)$ and the solution of Eq. (6) also changes as

$$
\begin{aligned}
& \widetilde{\alpha}_{1, k}=\widetilde{\alpha}_{1,0}+\widetilde{\alpha}^{\prime}=\frac{d \widetilde{\alpha}_{0}}{d \widetilde{\zeta}}+\widetilde{\alpha}^{\prime}, \\
& \widetilde{\beta}_{1, k}=\widetilde{\beta}_{1,0}+\widetilde{\beta}^{\prime}=\frac{d \widetilde{\beta}_{0}}{d \widetilde{\zeta}}+\widetilde{\beta}^{\prime},
\end{aligned}
$$

where $\widetilde{\alpha}^{\prime}$ and $\widetilde{\beta}^{\prime}$ are of $O(\epsilon)$ as well. Following the substitution of Eq. (10) into Eq. (6), the zeroth-order terms in $\epsilon$ return the solution for $k=0$, while the first-order terms yield

$$
\left(\begin{array}{cc}
\omega+\delta^{\prime} k^{2} & 0 \\
0 & \beta_{s} \omega+k^{2}
\end{array}\right)\left(\begin{array}{c}
\frac{d \widetilde{\alpha}_{0}}{d \widetilde{\zeta}} \\
\frac{d \widetilde{\beta}_{0}}{d \widetilde{\zeta}}
\end{array}\right)=\hat{L}\left(\begin{array}{c}
\widetilde{\alpha}^{\prime} \\
\widetilde{\beta}^{\prime}
\end{array}\right) .
$$

Since $\hat{L}$ has a zero eigenvalue, the solvability condition of Eq. (11) (Ref. 20) leads to 


$$
\int_{-\infty}^{+\infty}\left(\begin{array}{l}
\psi_{1} \\
\psi_{2}
\end{array}\right)^{T}\left(\begin{array}{cc}
\omega+\delta^{\prime} k^{2} & 0 \\
0 & \beta_{s} \omega+k^{2}
\end{array}\right)\left(\begin{array}{c}
\frac{d \widetilde{\alpha}_{0}}{d \widetilde{\zeta}} \\
\frac{d \widetilde{\beta}_{0}}{d \widetilde{\zeta}}
\end{array}\right) d \widetilde{\zeta}=0
$$

where $\psi_{1}$ and $\psi_{2}$ are the components of the right zero eigenvector of the adjoint matrix operator

$$
\hat{L}^{*}=\left(\begin{array}{cc}
\delta^{\prime} \frac{\partial^{2}}{\partial \widetilde{\zeta}^{2}}-Q_{0}^{\prime} \frac{\partial}{\partial \widetilde{\zeta}}-\widetilde{\beta}_{0}^{2} & \widetilde{\beta}_{0}^{2} \\
-2 \widetilde{\alpha}_{0} \widetilde{\beta}_{0} & \frac{\partial^{2}}{\partial \widetilde{\zeta}^{2}}-Q_{0}^{\prime} \frac{\partial}{\partial \zeta}+2 \alpha_{0} \beta_{0}
\end{array}\right) .
$$

Rewriting Eq. (12) as

$$
\begin{aligned}
& \omega \int_{-\infty}^{+\infty}\left(\psi_{1} \frac{d \widetilde{\alpha}_{0}}{d \widetilde{\zeta}}+\beta_{s} \psi_{2} \frac{d \widetilde{\beta}_{0}}{d \widetilde{\zeta}}\right) d \widetilde{\zeta} \\
& =-k^{2} \int_{-\infty}^{+\infty}\left(\delta^{\prime} \psi_{1} \frac{d \widetilde{\alpha}_{0}}{d \widetilde{\zeta}}+\psi_{2} \frac{d \widetilde{\beta}_{0}}{d \widetilde{\zeta}}\right) d \widetilde{\zeta},
\end{aligned}
$$

where the integral on the left-hand side may be set to unity with proper normalization of the eigenvector, leads to

$$
\left.\frac{d \omega}{d\left(k^{2}\right)}\right|_{k=0}=-\int_{-\infty}^{+\infty}\left(\delta^{\prime} \psi_{1} \frac{d \widetilde{\alpha}_{0}}{d \widetilde{\zeta}}+\psi_{2} \frac{d \widetilde{\beta}_{0}}{d \widetilde{\zeta}}\right) d \widetilde{\zeta} .
$$

Planar fronts at various $\varepsilon$ therefore lose stability when the integral in Eq. (15) becomes negative as $\delta^{\prime}$ exceeds some critical value which implies that in the original parameters, the instability threshold can be written as $\delta_{\mathrm{cr}}=\delta_{\mathrm{cr}}^{\prime} \beta_{s}$. Moreover, we see that there is a single $\delta_{\text {cr }}^{\prime}$ for the onset of instability because in Eq. (9) $\delta^{\prime} \rightarrow \delta$ and $Q_{0}^{\prime} \rightarrow c$ as $\varepsilon \rightarrow 0$. Hence the condition in Eq. (15) leads to that applied by Kapral and co-workers ${ }^{4}$ for reaction-diffusion fronts in the absence of drift $\left(\delta_{\mathrm{cr}}=2.300\right.$ at $\left.\varepsilon=0\right)$.

\section{NUMERICAL STUDY}

\section{A. Region of instability}

The one-dimensional front profile governed by Eq. (2) is represented by a heteroclinic orbit in the $(\alpha, \beta, d \beta / d \zeta)$ phase space connecting the two steady states corresponding to the boundary conditions. ${ }^{21}$ By applying a standard shooting method using the CVODE package, ${ }^{22}$ we select velocities for which the trajectory leaving the state at $\zeta=-\infty$ along the unstable manifold approaches the state at $\zeta=+\infty$ along the stable manifold. The upper branch of the solution contains the minimum velocities generally selected by onedimensional stable fronts propagating into the unstable state, ${ }^{18}$ and the turning point at the end of this branch represents the limit for a reaction-front solution. ${ }^{23}$ For the determination of $\delta_{\mathrm{cr}}^{\prime}$, the front profile is taken from the final shooting and the eigenvector of the adjoint matrix operator $\hat{L}^{*}$ is calculated from

$$
0=\hat{L}^{*}\left(\begin{array}{l}
\psi_{1} \\
\psi_{2}
\end{array}\right),
$$

with a relaxation technique ${ }^{24}$ as all other modes decay rapidly, leaving the desired eigenvector after a transient time. ${ }^{4}$ The integration is carried out using the CVODE package on 8001 points with a spacing of $\Delta \widetilde{\zeta}=0.05$. The onset of instability is determined by varying $\delta^{\prime}$ iteratively with a maximum tolerance of $10^{-6}$ for the integral in Eq. (15).

\section{B. Dispersion relation}

The dispersion relation may simply be calculated by taking a new form for the perturbation $\left[\phi_{k} \widetilde{\alpha}_{1, k}\right.$ $\left.=\varphi_{\alpha} \exp (i k \widetilde{\eta}), \phi_{k} \widetilde{\beta}_{1, k}=\varphi_{\beta} \exp (i k \widetilde{\eta})\right]$ from

$$
\left(\begin{array}{c}
\frac{\partial \varphi_{\alpha}}{\partial \widetilde{\tau}} \\
\beta_{s} \frac{\partial \varphi_{\beta}}{\partial \widetilde{\tau}}
\end{array}\right)=\hat{L}\left(\begin{array}{c}
\varphi_{\alpha} \\
\varphi_{\beta}
\end{array}\right)+\left(\begin{array}{cc}
\delta^{\prime} k^{2} & 0 \\
0 & k^{2}
\end{array}\right)\left(\begin{array}{l}
\varphi_{\alpha} \\
\varphi_{\beta}
\end{array}\right),
$$

a version of Eq. (6). After a transient period, $\left(\partial \varphi_{\alpha} / \partial \widetilde{\tau}\right) / \varphi_{\alpha}$ and $\left(\partial \varphi_{\beta} / \partial \widetilde{\tau}\right) / \varphi_{\beta}$ become constant, yielding $\omega$ within a set error of $0.1 \%$. As $k$ is increased, the initial condition is taken as the mode obtained in the previous run, using the same method as that applied for calculating $\psi_{1}$ and $\psi_{2}$. The results are transformed into the unscaled coordinate system of Eq. (1) as $\omega \beta_{s}^{2} \rightarrow \omega$ and $k \sqrt{\beta_{s}} \rightarrow k$, and compared to those obtained from the direct integration of Eq. (1) applying an operator-splitting method with a Crank-Nicholson scheme ${ }^{24}$ on a $501 \times 401$ grid with a spacing of 0.9 , a time step of 0.01 , and Neumann boundary conditions. For initial conditions, the planar boundary separating the two steady states is perturbed by shifting one randomly chosen grid line by one point in the direction of propagation. During the calculations the grid is adjusted in order to keep the front in the center. The coefficients of the Fourier cosine series associated with the front position-defined as the location of maximum reaction rate along $\zeta$-are determined at given intervals, the initial time dependence of which yields $\omega$ for the various modes.

\section{RESULTS AND DISCUSSION}

When the drift caused by electric field tends to separate the components, i.e., $\Delta \mathrm{v}<0$, a critical field strength $\varepsilon_{\mathrm{cr}}$ exists, beyond which reaction fronts fall apart and give rise to two independent electrophoretic fronts spreading with increasing distance between them. For the systematic study with reactant $A$ diffusing slightly faster than autocatalyst $B \quad(1 \leqslant \delta<3)$, we have chosen pairs of charges so that separation occurs at negative field, ${ }^{25}$ the result of which may readily be transfered to opposite charges by a simple change of signs. For the onset of instability $\delta_{\mathrm{cr}}^{\prime}=2.3002$ in accordance with the results of Kapral and co-workers in the absence of drift, and hence the critical ratio of diffusion coefficients is given as $\delta_{\mathrm{cr}}=2.3002 \beta_{s}$. The planar front remains stable for equal diffusion coefficients because the minimum of $\delta_{\text {cr }}$ is slightly above unity as shown in Fig. 1. Since the concentration of the autocatalyst behind the front $\beta_{s}$ is solely a function of the ratio of diffusion coefficients $\delta$ and the 


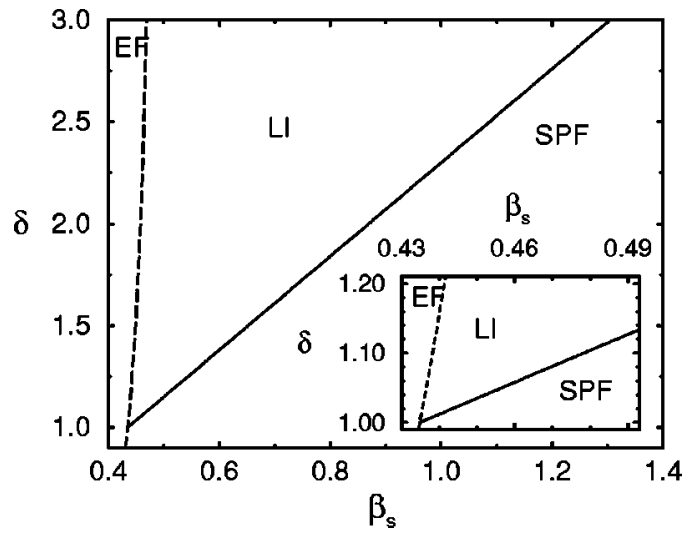

FIG. 1. Phase diagram showing the regions of stable planar reaction fronts $(\mathrm{SPF})$; lateral instability (LI); electrophoretic fronts $(\mathrm{EF})$ in the $\left(\delta, \beta_{s}\right)$-plane. Curves calculated from the linear stability analysis represent the onset of instability with solid line $\left(\delta_{\mathrm{cr}}=2.3002 \beta_{s}\right)$ and the extinction of reaction-diffusion fronts with dashed line.

difference in the drift velocity of the components $\Delta \mathrm{v}$, the region of stability is presented in the $(\delta, \Delta \mathrm{v})$-plane for all charges in Fig. 2 utilizing the relationship

$$
\Delta \mathrm{v}=\beta_{s}^{1 / 2}\left(\beta_{s}-1\right) c_{\varepsilon=0},
$$

obtained from the definition of $Q_{0}^{\prime}$ and Eq. (3) with $c_{\varepsilon=0}$ $=0.590147$ for the onset of instability. Planar fronts lose stability as the separation of components is increased, i.e., $\Delta \mathrm{v}$ is decreased, resulting in the formation of cellular structures. Further decrease in the difference of drift velocities leads to the extinction of reaction fronts. Figure 2 also shows that the critical ratio of diffusion coefficients $\delta_{\text {cr }}$ can be substantially decreased by approaching $\varepsilon_{\mathrm{cr}}$.

The dispersion relation is illustrated for an example case above and below the onset of instability in Fig. 3. The temporal eigenvalues are in good agreement with those of the individual modes present in the initial growth or decay of a small random perturbation obtained from the direct integration of Eq. (1). The calculation of $\omega(k)$ at $\delta=5.0$, i.e., farther from the onset of instability, indicates that even though planar fronts become more unstable on approaching $\varepsilon_{\mathrm{cr}}$, the

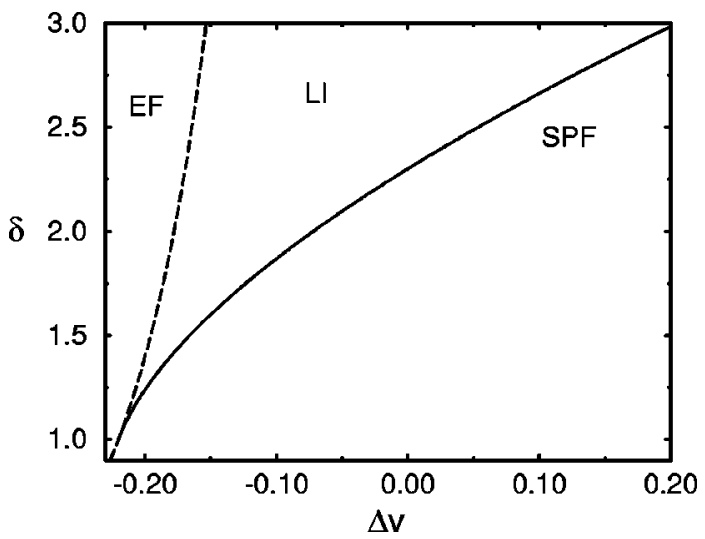

FIG. 2. Phase diagram showing the regions of stable planar reaction fronts (SPF); lateral instability (LI); electrophoretic fronts (EF) in the $(\delta, \Delta \mathrm{v})$-plane. Curves calculated from the linear stability analysis represent the onset of instability with solid line and the extinction of reactiondiffusion fronts with dashed line.

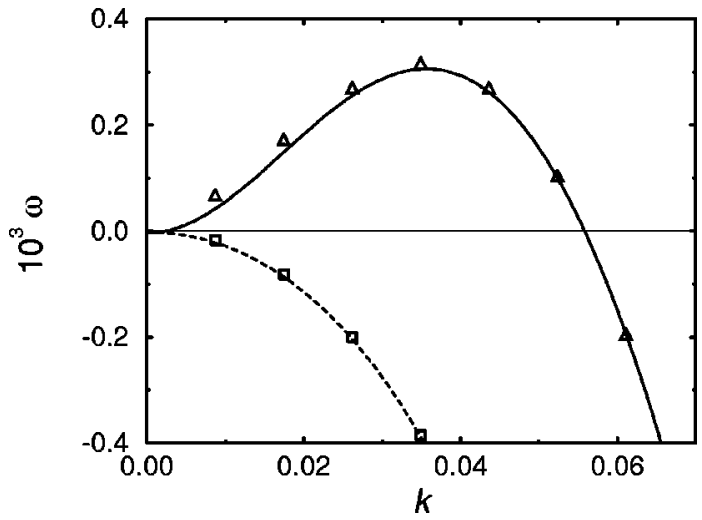

FIG. 3. Dispersion relation in the unscaled coordinate system for an example case of $z_{A}=-1, z_{B}=+1, \varepsilon=-0.08$. Curves from the linear stability analysis are drawn with solid line for $\delta=1.4$ and dashed line for $\delta=1.3$. Symbols represent the eigenvalues of the Fourier modes associated with the front evolved from a perturbed planar front in the full 2D problem with $\triangle$ for $\delta=1.4$ and $\square$ for $\delta=1.3$.

range of $k$ for growing modes in the unscaled coordinate system shrinks because of the decrease in $\beta_{s}$. This is in accordance with our earlier results on integrating Eq. (1); not only the amplitude of cellular structure but also the individual cell size increases towards $\varepsilon_{\mathrm{cr}} \cdot{ }^{17}$

When we plot the region of stability as a function of electric field strength for a given pair of charges, two distinct scenarios arise; the relation of the mobilities remains unchanged or is reversed as $\delta$ is increased. The former resembles the general picture in Fig. 2, while the latter reveals a division of the region of instability as a result of the unconditional stability of planar fronts for all $\varepsilon$ at equal mobilities, as shown in Fig. 4.

\section{CONCLUSION}

We have determined the region of lateral instability accurately by applying a linear stability analysis on the full
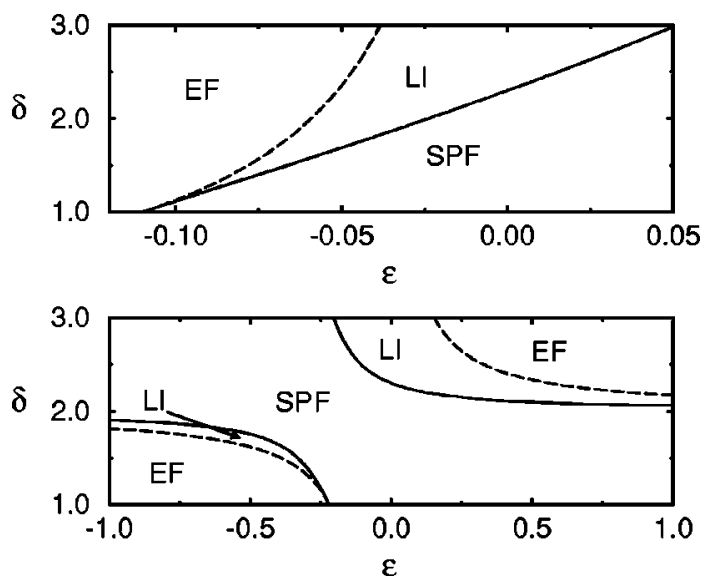

FIG. 4. Typical diagram showing the regions of stable planar reaction fronts $(\mathrm{SPF})$; lateral instability $(\mathrm{LI})$; electrophoretic fronts $(\mathrm{EF})$ in the $(\delta, \varepsilon)$-plane with the relation of mobilities unchanged (top), and reversed at $\delta=2$ (bottom). Curves from the linear stability analysis represent the onset of instability with solid line and the extinction of reaction-diffusion fronts with dashed line. The corresponding charges are $z_{A}=-1, z_{B}=+1$ (top) and $z_{A}$ $=1, z_{B}=2$ (bottom). 
reaction-diffusion system with advective terms. An appropriate scaling has elucidated that the critical ratio of the diffusion coefficients for any field strength can be derived from that in the absence of electric field. The results confirm that the thin-reaction-front approximation ${ }^{17}$ not only reveals the importance of the concentration of the autocatalyst behind the front for the stability of planar fronts but also predicts the existing proportionality between $\delta_{\text {cr }}$ and $\beta_{s}$. By varying the drift of components, the critical ratio of diffusion coefficients may be substantially varied with the region of lateral instability lying between that of the stable planar reaction fronts and the electrophoretic fronts for $\delta>1$. The analysis has also led to the accurate determination of the dispersion relation which shows that the drift caused by the electric field sharply influences the number of unstable modes initially present beyond the onset of instability.

\section{ACKNOWLEDGMENTS}

This work was supported by the Hungarian Science Foundation (F 022037). D.H. would like to thank Professor Y. Nishiura for his valuable comments. W.v.S. is grateful to Professor Nishiura for an invitation to his group and his hospitality. The present collaboration is an outgrowth of this visit.

${ }^{1}$ Y. Kuramoto, in Dynamics of Synergetic Systems, edited by H. Haken (Springer, Berlin, 1980), p. 134.

${ }^{2}$ D. Horváth, V. Petrov, S. K. Scott, and K. Showalter, J. Chem. Phys. 98, 6332 (1993).
${ }^{3}$ R. A. Milton and S. K. Scott, Proc. R. Soc. London, Ser. A 452, 391 (1996).

${ }^{4}$ A. Malevanets, A. Careta, and R. Kapral, Phys. Rev. E 52, 4724 (1995).

${ }^{5}$ D. Horváth and K. Showalter, J. Chem. Phys. 102, 2471 (1995).

${ }^{6}$ Á. Tóth, I. Lagzi, and D. Horváth, J. Phys. Chem. 100, 14837 (1996).

${ }^{7}$ D. Horváth and Á. Tóth, J. Chem. Phys. 108, 1447 (1998).

${ }^{8}$ Á. Tóth, B. Veisz, and D. Horváth, J. Phys. Chem. A 102, 5157 (1998).

${ }^{9}$ H. Ševčíková, M. Marek, and S. C. Müller, Science 257, 951 (1992).

${ }^{10}$ O. Steinbock, J. Schütze, and S. C. Müller, Phys. Rev. Lett. 68, 248 (1992).

${ }^{11}$ H. Ševč́ková and M. Marek, Physica D 13, 379 (1984).

${ }^{12}$ M. Watzl and A. F. Münster, J. Phys. Chem. A 102, 2540 (1998).

${ }^{13}$ A. B. Rovinsky and M. Menzinger, Phys. Rev. Lett. 69, 1193 (1992).

${ }^{14}$ A. B. Rovinsky and M. Menzinger, Phys. Rev. Lett. 70, 778 (1993).

${ }^{15}$ A. B. Rovinsky, A. M. Zhabotinsky, and I. R. Epstein, Phys. Rev. E 58, 5541 (1998).

${ }^{16}$ G. I. Sivashinsky, Combust. Sci. Technol. 15, 137 (1977).

${ }^{17}$ D. Horváth, Á. Tóth, and K. Yoshikawa, J. Chem. Phys. 111, 10 (1999).

${ }^{18}$ J. H. Merkin, H. Ševćíková, D. Šnita, and M. Marek, IMA J. Appl. Math. 60, 1 (1998)

${ }^{19}$ Y. Kuramoto, Chemical Oscillations, Waves, and Turbulence (Springer, Berlin, 1984).

${ }^{20}$ See, for example, P. Grindrod, Patterns and Waves (Clarendon, Oxford, 1991), pp. 22-23.

${ }^{21}$ J. Billingham and D. J. Needham, Philos. Trans. R. Soc. London, Ser. A 334, 1 (1991).

${ }^{22}$ S. D. Cohen and A. C. Hindmarsh, Comput. Phys. 10, 138 (1996).

${ }^{23}$ While this is well known in the literature of reaction-diffusion equations, it actually holds much more generally. See, e.g., U. Ebed and W. van Saarloos, Physica D (accepted).

${ }^{24}$ See, for example, W. H. Press, S. A. Teukolsky, W. T. Vetterling, and B. P. Flannery, Numerical Recipes in C (Cambridge University Press, Cambridge, 1992).

${ }^{25}$ The set of charges $\left(z_{A}, z_{B}\right)$ selected for the calculations, $-3,-1 ;-2,-1$; $-2,0 ;-1,-1 ;-1,0 ;-1,+1 ; 0,+1 ; 0,+2 ;+1,+2$. 